\title{
Managing psychosis in Parkinson's disease without drugs
}
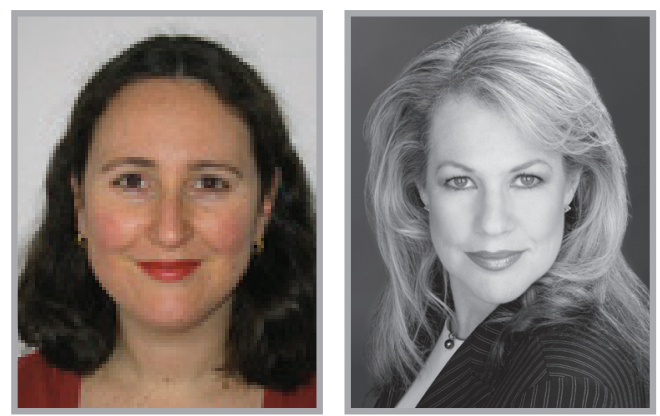

Anne PF Wand ${ }^{* 1,2,3}$ \& Carmelle Peisah ${ }^{2,4}$

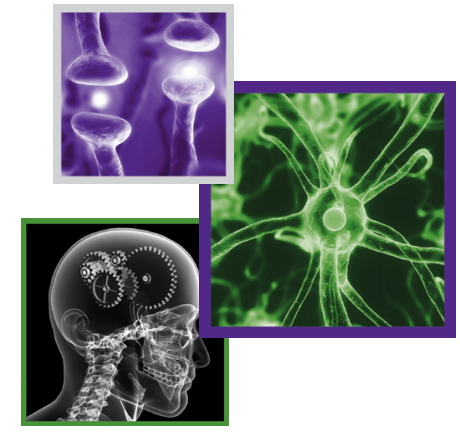

"Optimizing extrinsic factors such as the environment and personalizing approaches to individuals make intuitive sense ... lack of scientific evidence for psychosocial interventions should not prevent clinicians pursuing individualized person-centered psychosocial care.”

Psychosis in Parkinson's disease (PD) is common and the associated poor outcomes mandate a rigorous approach to identification and treatment. Psychosis is associated with depressive symptoms, poor quality of life, impaired activities of daily living, increased caregiver burden, nursing home placement and mortality [1-4]. Although drug treatments have been extensively investigated and reviewed, there has been a paucity of discourse on nondrug treatments, despite the complexities and risks of drug-based treatments in this population [5]. In this editorial, we briefly explore nonpharmacological treatment options and, in the absence of a substantial empirical evidence base, we draw lessons from the dementia and schizophrenia literature.

The nature \& prevalence of psychosis in PD

Psychosis, not typically considered a primary symptom of $\mathrm{PD}$, was rarely reported prior to the use of levodopa [6], and was thus considered a consequence of dopaminergic medications and dementia [7]. However, recent studies suggesting that dopaminergic treatment per se is insufficient to cause PD psychosis and the lack of dose-symptom relationship suggests a more complex etiology probably caused by the complex interplay of extrinsic and intrinsic diseaserelated factors $[1,9,10]$. Additionally, rates of psychosis in community samples of people with PD without dementia may be as high as $30 \%[1,8]$. Overall, psychotic symptoms occur in $20-40 \%$ of people with PD, with visual hallucinations occurring most commonly, followed by auditory hallucinations and delusions [7,11]. 'Minor symptoms,' such as passage hallucinations, illusions and sense of presence are less often studied but may be particularly prevalent $[1,8]$.

\section{Assessment of psychotic symptoms \\ in PD \\ The differential diagnosis of psychosis in PD is broad and includes delirium and dementia, psychiatric disorders, substance}

\section{KEYWORDS}

dementia $\bullet$ medication $\bullet$ Parkinson's disease $\bullet$ pharmacotherapy

- psychosis • psychosocial

- psychotherapy

'Older Persons' Mental Health Service, St George Hospital, Rear Demountable, 7 Chapel St, Kogarah, NSW 2217,

Australia

${ }^{2}$ Faculty of Medicine, University of New South Wales, Sydney, Australia

${ }^{3}$ South Eastern Sydney Local Health District, Sydney, Australia

${ }^{4}$ Sydney Medical School, University of Sydney, Sydney, Australia

*Author for correspondence: Tel.: +61 29113 2035; a.wand@unsw.edu.au

Future 
misuse or withdrawal and pharmacotherapy. Thus, the first step in management should involve excluding and treating potentially reversible causes. Delirium, which is common in PD especially among those with comorbid cognitive impairment, must be considered with any acute decline in cognition, function or mental state. A medical assessment (including review of potentially deliriogenic medication, especially drugs with anticholinergic effects, e.g., benzhexol, benztropine) targeted physical examination and broad screening investigations (including electrolytes, renal, thyroid and liver function, full blood count, urinalysis, C-reactive protein, CXR etc.) as well as those specific to the patient's medical history (e.g., ECG, EEG, neuroimaging etc.) is required. The underlying cause should then be treated and the patient monitored over time in an appropriate setting to ensure resolution of the delirium.

Psychotic symptoms in PD may also occur secondarily to mental illnesses such as schizophrenia, depression, mania and dementia, including other Parkinsonian syndromes such as dementia with Lewy bodies. A careful history taken from the patient and the family will aid clarification of the underlying diagnosis. Extrinsic, drug-related factors are perhaps the most remediable causes of psychosis in PD. Accordingly, polypharmacy, a risk factor for psychosis in PD per se [12] should be addressed, as should psychoactive substance misuse and withdrawal, especially amphetamines, cannabis, cocaine and alcohol. Dopaminergic medications which may cause or worsen psychotic symptoms [11], should be reduced to the minimum dose and total number of agents withdrawn in a step-wise manner, starting with those with the highest risk-to-benefit ratio. Therefore, anticholinergics should be weaned and ceased first followed by selegiline (monoamine oxidase B inhibitor) and amantadine (glutamate agonist), then dopamine agonists (such as pramipexole, bromocriptine), then catechol-O-methyltransferase inhibitors (e.g., entacapone) and lastly levodopa/carbidopa [11]. The aim is to strike a balance between improving drug-related psychotic symptoms while not significantly worsening the motor symptoms of PD.

\section{Specific nonpharmacological strategies for psychosis in PD}

After excluding organic and psychiatric illness and rationalizing PD medication, a number of other simple strategies may be helpful in addressing psychotic symptoms, although some symptoms may be easier to treat nonpharmacologically than others. In light of some of the deficits in visual processing, acuity, color and contrast recognition and ocular pathology associated with visual hallucinations, correcting remediable visual problems and optimizing the environment may be helpful in reducing visual misinterpretation. In the early stages of psychosis, patients tend to have insight into their symptoms, and may be amenable to reality testing that what they are seeing (or hearing) is not actually there. Diederich and colleagues in an observational study reported that 36 out of 46 visually hallucinating PD patients (including nine with dementia) found self-initiated coping strategies such as cognitive, interactive and visual techniques helpful in managing symptoms [13]. Cognitive strategies involved self-talk/ reassurance that the hallucinations are not real, and actions to extinguish hallucinations (such as turning on a light). Interactive techniques engaged the carer/family in discussion to realitytest hallucinations and seek reassurance. Visual techniques include focusing better upon the hallucination, looking away from it or focusing on another object.

In contrast, delusions in PD are usually of a common theme, often paranoid in nature and typically of spousal infidelity, theft, poisoning food or substitution of PD medications. Because they are paranoid in nature, they can be more threatening, possibly less amenable to nondrug treatments and more immediate action is often necessary.

\section{- Insights from the schizophrenia literature}

The review of current evidence-based psychosocial treatment interventions for persons with schizophrenia by the Schizophrenia Patient Outcomes Research Team produced treatment recommendations in a number of areas, albeit few with relevance specifically to the treatment of positive symptoms, which might share some commonality with PD psychosis. These included cognitive behavioral therapy (CBT), family-based interventions and assertive community treatment outreach [14]. Certainly the notion of CBT for PD is appealing given that early psychosis typically involves retained insight [10], which might maximize the response to typical CBT approaches in schizophrenia which include a focus on the patient's view of 
symptom or problems and the development of more rational- or adaptive-coping responses [14]. Although CBT has been investigated in the management of depression in PD, it has not been directly evaluated in PD psychosis [15].

Family interventions (in combination with antipsychotic therapy) in people with schizophrenia include psychoeducation, emotional support, crisis intervention and learning strategies to cope with symptoms of the illness and associated problems [14]. Given the relationship between psychosis and caregiver burden, because symptoms can be markedly disturbing for both patients and caregivers and make caregiving more challenging and overwhelming, interventions such as these must be a mandatory part of any treatment package.

\section{- Insights from the behavioral \& psychological symptoms of dementia literature}

Cognitive impairment and dementia are an expected part of the natural history of PD, with prevalence increasing with the duration of PD [9]. Although psychosis may occur in PD without dementia, it is more likely to happen in those with dementia [7], and insights can be gained from the treatment of behavioral and psychological symptoms of dementia, where it is imperative to try nonpharmacological options first [16]. Principles of management include person-centered care, optimization of sensory, communication and physical function and the environment, and behavioral strategies. Key nonpharmacological strategies with (mostly modest) evidence for psychosis in people with dementia which may be relevant to psychosis in PD include psychoeducation of caregivers, behavior management for patients and caregivers, cognitive stimulation therapy and validation therapy [16]. In the latter therapy, the person's feelings associated with the experience are validated as real. For example, if hallucinating the presence of a deceased loved one, the person is encouraged to explore their feelings and is engaged in a discussion about their relationship.

\section{Other nonpharmacological treatments for} psychosis in Parkinson's disease

Electroconvulsive therapy (ECT) and deepbrain stimulation (DBS) have been proposed as treatments for psychosis in PD. In general, studies are few, often case reports (summarized by Quelhas [15]) and of small sample size. It is not clear whether ECT is of benefit in PD with depression and/or psychosis [15]. One study of ECT in eight people with PD psychosis reported an attenuation of symptoms and the severity of PD after ECT [16]. Although DBS of the subthalamic nucleus and globus pallidus pars interna may have effects on cognitive impairment, there is no evidence that DBS improves psychotic symptoms [17]. Indeed, case reports describe new onset of psychosis or hallucinations postoperatively, albeit rarely and mostly transiently [18,19].

\section{Conclusion}

Any patient with PD presenting with psychotic symptoms must be carefully assessed to exclude potentially reversible causes such as delirium, a primary mental illness and sensory impairment, or dementia. Thereafter, there is a hierarchy of PD medications to be withdrawn to reach a balance between acceptable motor function and psychotic symptoms. Antipsychotic medication for psychosis in $\mathrm{PD}$ is problematic due to high side-effect burden, effects on the motor symptoms of PD and lack of good evidence of efficacy. Therefore, nonpharmacological approaches should be considered. To date, there are few studies directly evaluating the efficacy of such strategies in PD, although it is possible to extrapolate from some of the psychosocial strategies used to manage behavioral \& psychological symptoms of dementia or residual psychotic symptoms in schizophrenia, such as family support and cognitive behavioral techniques. Optimizing extrinsic factors such as the environment and personalizing approaches to individuals make intuitive sense. Urgent research is needed to evaluate nonpharmacological approaches to treating psychosis in PD. However, the current lack of scientific evidence for psychosocial interventions should not prevent clinicians pursuing individualized person-centered psychosocial care.

\section{Financial \& competing interest disclosure}

The authors have no relevant affliations or financial involvement with any organization or entity with a financial interest in or financial conflict with the subject matter or materials discussed in the manuscript. This includes employment, consultancies, honoraria, stock ownership or options, expert testimony, grants or patents received or pending, or royalties.

No writing assistance was utilized in the production of this manuscript. 


\section{References}

1 Mack JL, Rabins PV, Anderson K et al. Prevalence of psychotic symptoms in a community-based Parkinson's disease sample. Am. J. Geriatr. Psychiatry 20, 123-131 (2012).

2 Aarsland D, Larsen JP, Tandbert E. Predictors of nursing home placement in PD: a population-based prospective study. J. Am. Geriatr. Soc. 48, 938-942 (2000).

3 Goetz CG, Stebbins GT. Risk factors for nursing home placement in advanced Parkinson's disease. Neurology 43,2227-2229 (1993).

4 Ravina B, Marder K, Fernandez HH et al. Diagnostic criteria for psychosis in Parkinson's disease: report of an NINDS/ NIMH work group. Mov. Disord. 22(8),1061-1068 (2007)

5 Seppi K, Weintraub D, Coelho M et al. The movement disorder society evidence-based medicine review update: treatments for the non-motor symptoms of Parkinson's disease. Mov. Disord. 26 Suppl 3, S42-S80 (2011).

6 Fenelon G, Goetz CG, Karenberg A. Hallucinations in Parkinson disease in the prelevodopa era. Neurology 66, 93-98 (2006).
7 Grover S, Somaiya M, Kumar S, Avasthi A Psychiatric aspects of Parkinson's disease. J. of Neurosci. in Rural Pract. 6, 65-76 (2015).

8 Rabey JM. Hallucinations and psychosis in Parkinson's disease. Parkinsonism Relat. Disord. Suppl 4,S105-S110 (2009).

9 Hindle JV. The practical management of cognitive impairment and psychosis in the older Parkinson's disease patient. J. Neural Transm. 120, 649-653 (2013).

10 Zahodne LB, Fernandez HH. A review of the pathophysiology and treatment of psychosis in Parkinson's disease. Drugs Aging 25(8), 665-682 (2008)

11 Aarons S, Peisah C, Wijeratne C. Neuropsychiatric effects of Parkinson's disease treatment. Australas. J. Ageing 31, 198-202 (2012).

12 Henderson MJ, Mellers JDC. Psychosis in Parkinson's disease: "between a rock and a hard place”. Int. Rev. Psychiatry 12, 319-334 (2000).

13 Diederich NJ, Pieri V, Goetz CG. Coping strategies for visual hallucinations in Parkinson's disease. Mov. Disord. 18(7), 831-838 (2003).
14 Dixon LB, Dickerson F, Bellack AS et al. The 2009 schizophrenia PORT psychosocial treatment recommendations and summary statements. Schizophr. Bull. 36(1), 48-70 (2010).

15 Quelhas R. Psychiatric care in Parkinson's disease. J. of Psychiatric Practice 19, 118-141 (2013).

16 Draper B, Wand APF. Behavioural and psychological symptoms of dementia. In: Encyclopedia of Geropsychology. Pachana N (Ed.). (2015) (In Press).

17 Williams-Gray CH, Foltynie T, Lewis SJG, Barker RA. Cognitive deficits and psychosis in Parkinson's disease. A review of the pathophysiology and therapeutic options. CNS Drugs 20, 477-505 (2006).

18 Anderson KE, Mullins J. Behavioural changes associated with deep brain stimulation surgery for Parkinson's disease. Curr. Neurol. Neurosci. Rep. 3(4), 306-313 (2003).

19 Funkiewiez A, Ardouin C, Caputo E et al. Long term effects of bilateral subthalamic nucleus stimulation on cognitive function, mood, and behaviour in Parkinson's disease. J. Neurol. Neurosurg. Psychiatry 75(6), 834-839 (2004) 